\title{
CO-INFECTION OF PLASMODIUM VIVAX AND FALCIPARUM MALARIA IN A TERTIARY CARE HOSPITAL IN BIHAR
}

\author{
Nag Sanjay1, Kumar Ajay², Lal Arjun³, Rajpal Kamlesh4, Mahto S. P5 \\ ${ }_{1}^{1}$ Assistant Professor, Department of Microbiology, Anugrah Narayan Magadh Medical College (ANMMC), Gaya. \\ ${ }^{2}$ Senior Resident, Department of Microbiology, Indira Gandhi Institute of Medical Sciences (IGIMS), Patna. \\ ${ }_{3}^{3}$ Assistant Professor, Department of Microbiology, Anugrah Narayan Magadh Medical College (ANMMC), Gaya. \\ ${ }^{4}$ Assistant Professor, Department of Microbiology, Anugrah Narayan Magadh Medical College (ANMMC), Gaya. \\ 5 Professor, Department of Microbiology, Anugrah Narayan Magadh Medical College (ANMMC), Gaya.
}

\begin{abstract}
BACKGROUND

Malaria is probably one of the oldest diseases known to mankind that has had profound impact on our history. It continues to be a huge social, economic and health problem, particularly in the tropical countries. Studies on malaria due to co-existent P. falciparum and P. vivax infections are negligible in India. Therefore, this study was undertaken to find out the clinical profile, progn ostic factors and outcome of mixed-species malaria and to compare it with P. falciparum malaria.
\end{abstract}

\section{MATERIALS AND METHODS}

A descriptive study was carried out on 100 patients of malaria admitted in Department of Medicine, Patna Medical College and Hospital, Patna, a tertiary care hospital from January 2016 to August 2017.

\section{RESULTS}

Out of 100 cases, 5 cases have both falciparum and vivax co-infection in infective positive cases. In 68 cases $P$. falciparum were positive and 27 were $P$. vivax positive.

\section{CONCLUSION}

Co-infection is not uncommon in the locality, where both species coexists. Co-infection can complicate with severe malaria. However, its incidence and severity is less than severe falciparum malaria. In mixed infection, $P$. vivax malaria has a protective effect against the severity of falciparum malaria.

\section{KEYWORDS}

Prevalence, Co-Infection, P. falciparum, P. vivax.

HOW TO CITE THIS ARTICLE: Sanjay N, Ajay K, Arjun L, et al. Co-infection of plasmodium vivax and falciparum malaria in a tertiary care hospital in Bihar. J. Evolution Med. Dent. Sci. 2017;6(95):6990-6992, DOI: 10.14260/jemds/2017/1517

\section{BACKGROUND}

Malaria was linked with poisonous vapours of swamps or stagnant water on the ground since time immemorial. This probable relationship was so firmly established that it gave the two most frequently used names to the disease malaria, later shortened to one word malaria (paludisme). ${ }^{1}$ Malaria is a protozoal disease transmitted by the bite of infected anopheles mosquitoes. It is the most important of the parasitic diseases of humans with transmission in 107 countries containing 3 billion people and causing 1 - 3 million death each year. Malaria is a major cause of mortality and morbidity in the tropical and subtropical regions of the world. Developed countries are relatively free of malaria, but it remains well entrenched across the tropical world. ${ }^{2}$

The incidence of malaria worldwide is estimated to be 300 - 500 million clinical cases each year with about 90 percent of these occurring in Sub-Saharan Africa, and mostly caused by

'Financial or Other Competing Interest': None.

Submission 22-09-2017, Peer Review 04-12-2017,

Acceptance 11-12-2017, Published 23-12-2017.

Corresponding Author:

Dr. Kumar Ajay,

Senior Resident,

Department of Microbiology,

Indira Gandhi Institute of Medical Sciences (IGIMS),

Patna-14.

E-mail:dr.ajay876@gmail.com

DOI: $10.14260 /$ jemds $/ 2017 / 1517$

\section{(c) (i) $(9)$}

P.falciparum. ${ }^{3}$ Of reported cases of malaria, India accounts for 77 percent of the regional total. Major endemic areas in India accounts for 77 percent of the regional total. Major endemic areas in India are in the North-Eastern states, Andhra Pradesh, Chhattisgarh, Gujarat, Jharkhand, Bihar, MP, Maharashtra, Rajasthan and Orissa. ${ }^{4}$

Five species of the genus Plasmodium cause nearly all malarial infections in humans. These are P. falciparum, $P$. vivax, $P$. ovale, $P$. malariae, and in Southeast Asia the monkey malaria parasite $P$. knowlesi, which can be reliably identified only by molecular methods. Almost all deaths are caused by falciparum malaria. Clinical pictures, outcome, prognostic factors and changing clinical pattern of malaria due to individual species infection have been studied extensively. ${ }^{1}$ In a geographical area when more than one species co-exist, sympatric combination of these infections in an individual cannot be ruled out. But the profile of malaria due to multiple species infection is considerably underestimated due to lack of studies. ${ }^{5}$

Human infection begins when a female anopheles mosquito inoculates plasmodium sporozoites from its salivary gland during a blood meal. These microscopic motile forms of the malarial parasite are carried rapidly via the bloodstream to the liver, where they invade hepatic parenchymal cells and begin a period of asexual reproduction. By this amplification process (known as intrahepatic or pre-erythrocytic schizogony or merogony), a single sporozoite eventually may produce from 10,000 to $>30,000$ daughter merozoites. The 
swollen infected liver cell eventually bursts, discharging motile merozoites into the bloodstream. These merozoites then invade the red blood cells (RBCs) and multiply six- to twenty-fold every $48-72 \mathrm{~h}$. When the parasites reach densities of 50/ L of blood (100 million parasites in the blood of an adult), the symptomatic stage of the infection begins. In P. vivax and $P$. ovale infections, a proportion of the intrahepatic forms do not divide immediately, but remain dormant for a period ranging from 3 weeks to a year or longer before reproduction begins. These dormant forms or hypnozoites are the cause of the relapses that characterise infection with these two species. ${ }^{6}$

Symptom of malaria include fever, shivering, arthralgia, vomiting, anaemia, haemolysis and jaundice, haemoglobinuria and convulsions. There may be a feeling of tingling in the skin, particularly with malaria caused by P. falciparum. ${ }^{7}$

The classical symptom of malaria is the cyclical occurrence of sudden coldness followed by rigor and then fever and sweating lasting for four to six hours, occurring every two days in $P$. vivax and $P$. ovale infections, while every three for $P$. malariae. P. falciparum can have recurrent fever every 36 - 48 hours or less pronounced and almost continuous fever. Splenomegaly, severe headache, cerebral ischaemia, hepatomegaly, hypoglycaemia and haemoglobinuria with renal failure may occur. ${ }^{8}$

Chronic malaria is seen in both P. vivax and P. ovale, but not in P. falciparum. Here, the disease can relapse months or years after exposure due to the presence of latent parasites in the liver. Describing a case of malaria as cured by observing the disappearance of parasites from the bloodstream can therefore be deceptive. ${ }^{9}$

If the patients with falciparum malaria have jaundice, vital organ complications should be looked for. Also, hyperpyrexia is no longer considered a sign of severity. 10

The diagnosis of malaria is essentially made from clinical features and can be confirmed by demonstration of plasmodium by laboratory examination. Light microscopy demonstrates different stages of malaria parasite. The expected sensitivity that for the examination of the thick blood film procedure is about 50 parasites/ $\mu \mathrm{L}$ of blood (assuming a total RBC count of $5 \times 106 / \mu \mathrm{L}$ of blood), which is equivalent to $0.001 \%$ of RBC infected. Milne et al found that most routine diagnostic laboratories generally achieved a lower sensitivity of detection (average $0.01 \%$ RBC infected and 500 parasites $/ \mu \mathrm{L}) .{ }^{11}$

Warhurst and Williams reported that examination of thin blood films is only $1 / 10$ as sensitive as examination of thick blood films for the quantification of malarial parasites, although morphological identification of the Plasmodium species present is much easier using thin films.

Certain fluorescent dyes have an affinity for the nucleic acid in the parasite nucleus and will attach to the nuclei. Two fluorochromes have frequently been used for this purpose, acridine orange (AO) and benzothiocarboxypurine (BCP). An alternative fluorochrome procedure using a solution of BCP reported sensitivity and specificity of $>95 \%$ for P.falciparum. ${ }^{12}$

In this context, the knowledge about mixed species infection is important not only for control measures, but also for therapeutic options and futuristic vaccine programme. Therefore, we have undertaken this research to study the clinical pattern and outcome of mixed species malaria in adults and to compare it with Pf malaria in a tertiary care hospital.

\section{MATERIALS AND METHODS}

A descriptive study was carried out on 100 patients of malaria admitted in Department of Medicine, Patna Medical College and Hospital, Patna, a tertiary care hospital from January 2016 to August 2017. Cases were selected with complaint of untreated fever of short duration and/ or raised body temperature without any preexisting documented systemic illness with clinical features like fever-paroxysmal, remittent or intermittent, chills, anaemia, splenomegaly, hepatomegaly, headache, vomiting, drowsiness, altered behaviour, confusion, unarousable coma etc. Confirmation of diagnosis was made by demonstration of plasmodium in the peripheral blood by thin and thick blood film examination, by immunochromatographic test, Quantitative Buffy coat analysis for parasite and complete blood profile included platelet.

\section{Statistical Analysis}

After confirming the diagnosis of malaria by peripheral blood smear examination and/ or immunochromatography and/ or Buffy coat examination, 100 cases were studied under various aspects as described in material and methods. The outcome of the study was statistically analysed, tabulated and compared.

\section{RESULTS}

Out of these 100 patients 68 cases had Plasmodium falciparum infection, 27 had malaria from P. vivax and 5 patients had coinfection of falciparum and vivax infection. Out of 100 cases studied, 77 were male and 23 were female. The age of patient varied from 13 - 75 years. The maximum incidence of the disease was observed among patients in the age group from 21 years to 40 years in both sexes [Diagram 1].

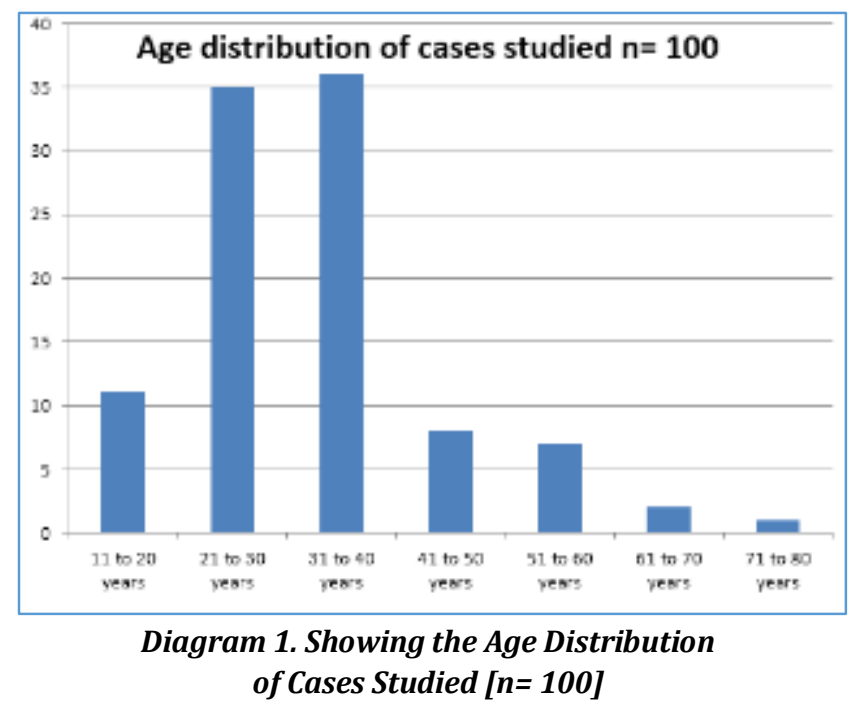

Out of 100 cases 62 were diagnosed by light microscopy in peripheral blood smears, 95 cases were diagnosed by immunochromatography [Optimal IT]. Out of these 100 patients 68 cases had Plasmodium falciparum infection, 27 had malaria from P. vivax and 5 patients had combined falciparum and vivax infection [Diagram 2, Table 1]. 


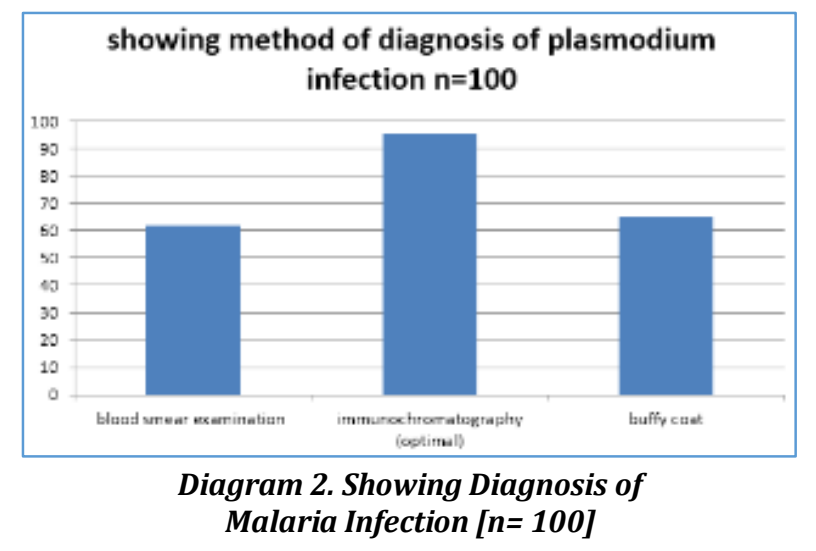

\begin{tabular}{|c|c|c|}
\hline Sl. No. & Plasmodium Species & No. of Cases \\
\hline 1. & P. falciparum & 68 \\
\hline 2. & P. vivax & 27 \\
\hline 3. & Combined (both falciparum and vivax) & 5 \\
\hline \multicolumn{2}{|c|}{ Table 1. Showing No. of Cases of Different Types of } \\
Plasmodium Infection (n= 100) \\
\hline \multicolumn{2}{|c|}{} \\
\hline
\end{tabular}

\section{DISCUSSION}

The present study was carried out in 100 patients of malaria admitted in Department of Medicine, Anugrah Narayan Magadh Medical College and Hospital, ${ }^{1}$ Gaya, a tertiary level hospital. The study period extended from January 2010 to August 2011. A total of 100 cases diagnosed as malaria and admitted in Medical Ward, Emergency and ICU were included for this study. 5 cases were diagnosed as having acute kidney injury as per RIFLE/ AKIN criteria in cases of malaria and comprising of different age groups ranging from 15 - 75 years and both sexes with male: female ratio of 3.0: 1. Besides fever (100\%), headache $(60 \%)$, vomiting (60\%), convulsion (36\%), unconsciousness (28\%) and abnormal behaviour (33\%) were other presenting complaints. Anaemia was present in $42 \%$, jaundice was noted in $46 \%$, hepatomegaly in $48 \%$ and splenomegaly in $86 \%$, pulmonary oedema/ ARDS in 3\%, diminished urine output in $24 \%$ and dark colour urine in $18 \%$. Investigations revealed TLC >12,000/ uL in $29 \%$, haemoglobin $<10 \mathrm{gm} \%$ in $59 \%$ and severe anaemia $(\mathrm{Hb} \%<5$ gm $\%$ ) in $22 \%$, serum bilirubin $>3 \mathrm{mg} / \mathrm{dL}$ in $44 \%$ and SGPT $>100 \mathrm{U} / \mathrm{L}$ in $30 \%$. Serum sodium was $<135 \mathrm{mEq} / \mathrm{L}$ in $41 \%$ cases and serum potassium was $>5.5 \mathrm{mEq} / \mathrm{L}$ in $22 \%$. Blood sugar level was $<40 \mathrm{gm} / \mathrm{dL}$ in $29 \%$. Severe anaemia and jaundice were common manifestations. Extrarenal manifestation with associated mortality in all the cases of malaria $(n=100)$ was as follows: Severe anaemia had $18.18 \%$ mortality, hyperbilirubinaemia had 15.9\% mortality, cerebral malaria had 25\% mortality, pulmonary oedema had 100\% mortality, hypoglycaemia had $31.03 \%$, hyponatraemia had $21.95 \%$ mortality and hyperkalaemia had $22.7 \%$ mortality. Pulmonary oedema/ ARDS had highest mortality; hypoglycaemia is the next. Quantitative Buffy coat is a laboratory test to detect infection with malaria or other blood parasites. 1 . The blood is taken in a QBC capillary tube, which is coated with Acridine Orange [a fluorescent dye] and centrifuged; 2 . The fluorescing parasite can then be observed under Ultraviolet light at the interface between RBC and Buffy coat.

\section{CONCLUSION}

In conclusion co-infection is not uncommon in the locality where both species coexist. Co-infection can complicate with severe malaria, but its incidence is significantly less suggesting a protective effect of $P$. vivax infection. It is important because if previous $P$. vivax infection does protect against severe $P$. falciparum infection, then control of vivax malaria may reduce the vivax-associated protection against severe falciparum infection enhancing increased incidence of severe falciparum malaria.

\section{REFERENCES}

[1] Abdalla S, Weatherall DJ, Wickramasinghe SN, et al. The anaemia of P. falciparum malaria". Br J Haematol 1980;46(2):171-83.

[2] Ahmad SH, Danish T, Faridi MM, et al. Renal function in acute malaria in children. J Trop Pediatr 1989;35(6):291-4.

[3] Mockenhaupt FP, Ehrhardt S, Burkhardt J, et al. Manifestation and outcome of severe malaria in children in northern Ghana. Am J Trop Med Hyg 2004;71(2):167-72.

[4] Thakur BB, Thakur S. Medicine update. Section 7, Chapter - 36, Vol 11. 2001:pp 241.

[5] Das BS. Renal failure in malaria. J Vector Borne Dis 2008;45(2):83-97.

[6] Bag S, Sarnal GC, Deep N, et al. Complicated falciparum malaria. Indian Paediatr 1994;31:821-5.

[7] Barsoum R, Sitprija V. Tropical nephrology. In: Disease of the Kidney. $6^{\text {th }}$ edn. Edited by Schrier RW, Gottaschalk CW, Boston, et al. Brown and Co, 1996:2221-68.

[8] Barsoum RS. Malarial nephropathies. Nephrol Dial Transplant 1998;13(6):1588-97.

[9] Beadle C, Long GW, Weiss WR, et al. Diagnosis of malaria by detection of plasmodium falciparum HRP-2 antigen with a rapid dipstick antigen-capture assay. Lancet 1994;343(8897):564-8.

[10] Berkley J, Mwarumba S, Bramharn K, et al. Bacteraemia complicating severe malaria in children. Trans R Soc Trop Med Hyg 1999;93(3):283-6.

[11] Bhamarapravati N, Boonpucknavig S, Boonpucknavig $\mathrm{V}$, et al. Glomerular changes in acute plasmodium infection. An immunological study. Arch Pathol 1973;96(5):289-93.

[12] Boonpucknavig V, Sitprija V. Renal disease in acute plasmodium falciparum infection in man. Kidney Int 1979;16(1):44-52. 